\title{
Electron mobility in Si delta doped GaAs
}

\author{
P.M. Koenraad ${ }^{\mathrm{a}, *}$, A.F.W. van de $\operatorname{Stadt}^{\mathrm{a}}$, J.M. Shi ${ }^{\mathrm{a}}$, G.Q. Hai ${ }^{\mathrm{b}}$, N. Studart ${ }^{\mathrm{b}}$, P. Vansant ${ }^{\mathrm{c}}$, \\ F.M. Peeters ${ }^{\mathrm{c}}$, J.T. Devreese ${ }^{\mathrm{a}, \mathrm{c}}$, J.A.A.J. Perenboom ${ }^{\mathrm{d}}$, J.H. Wolter ${ }^{\mathrm{a}}$

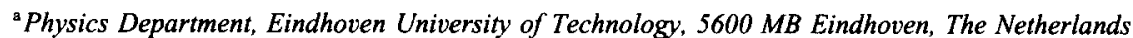 \\ b Physics Department, Federal University of São Carlos, 13565-905, SP, Brazil \\ 'Physics Department, University of Antwerp (UIA), Universiteitsplein 1, B-2610 Antwerpen, Belgium \\ ${ }^{\mathrm{d}}$ High-Field Magnet Laboratory, University of Nijmegen, 6525 ED Nijmegen. The Netherlands
}

\begin{abstract}
We have measured the transport and quantum mobility in $\mathrm{Si}$ delta doped samples as a function of the doping concentration and the thickness of the doping layer. The results are compared with mobility calculations which show that the ionized impurity scattering rate is determined by the fluctuations in the charge distribution of the delta layer instead of the full charge distribution itself.
\end{abstract}

\section{Introduction}

At low-temperatures ionized impurity scattering is one of the main scattering mechanisms in most semiconductor structures. Ionized impurity scattering has been studied extensively in GaAs/AlGaAs heterostructures. The strong improvement, in the last decade, of the mobility at low temperatures in $\mathrm{GaAs} / \mathrm{AlGaAs}$ heterostructures originated from the successful reduction of the ionized impurity scattering in these structures. In contrast with these high-mobility heterostructures are the well-known delta doping layers. In delta doping layers ionized impurity scattering is by far the most important scattering mechanism due to the strong overlap between the ionized dopant atoms and the free carriers. In delta doping layers normally more than one subband is populated. The population of multiple subbands will influence the scattering via screening and intersubband scattering. Thus delta doping layers are ideal structures for the study of ionized impurity scattering in the strong interaction

\footnotetext{
* Corresponding author.
}

regime, and for the study of the effect of multiple subband occupation on the ionized impurity scattering.

\section{Experimental methods and analysis}

We have studied ionized impurity scattering in delta doping layers by measuring both the transport and quantum mobility in $\mathrm{Si}$ delta doped $\mathrm{GaAs}$ layers. The quantum mobility and transport mobility in the case of a twodimensional electron gas are defined by the relations

$$
\begin{aligned}
& 1 / \mu_{\mathrm{q}}=\left(m^{*} / e\right) \int w(\theta) \mathrm{d} \theta, \\
& 1 / \mu_{\mathrm{t}}=\left(m^{*} / e\right) \int w(\theta)(1-\cos \theta) \mathrm{d} \theta,
\end{aligned}
$$

where $w(\theta)$ is the probability for scattering over an angle $\theta$. The quantum mobility is determined by the averaged scattering time, i.e. all scattering events have the same weight. The so-called transport mobility is determined mostly by the back-scattering events. The ratio of the 
transport to the quantum mobility thus gives information about the angle dependence, $W(\theta)$, of the scattering process. For instance for phonon scattering the scattering probability is isotropic and therefore the ratio is equal to 1. For ionized impurity scattering in $\mathrm{GaAs} / \mathrm{AlGaAs}$ heterostructures the ratio varies from 3 to 20 depending on the separation of the ionized impurities from the electron gas [1].

The transport mobility is obtained from the classical magneto-resistance in low magnetic fields. The classical magneto-conductivity is described by the Drude expressions

$\sigma_{x x}=\sum_{i} n_{i} \mu_{t, i} e /\left(1+\mu_{\mathrm{t}, i}^{2} B^{2}\right)$,

$\sigma_{x y}=\sum_{i} n_{i} \mu_{t, i}^{2} B e /\left(1+\mu_{t, i}^{2} B^{2}\right)$.

The quantum mobility is obtained from the amplitude dependence of the Schubnikov-de Haas $(\mathrm{SdH})$ oscillations in high magnetic fields. Typically a magneto-resistance measurement on a delta doped structure contains multiple oscillation periods where each period belongs to a populated subband. The individual oscillation periods are resolved by Fourier transformation. In the frequency domain we filter the peak corresponding to a single subband and perform an inverse Fourier transform to obtain the $\mathrm{SdH}$ oscillations of a single subband. The $\mathrm{SdH}$ oscillation amplitude of an individual subband is described by

$\Delta \rho_{x x}(:) X / \sinh (X) \exp \left(-\pi / \mu_{\mathrm{q}, 1} B\right) \cos (\pi h / e B-\pi)$,

where $X=4 \pi^{2} m^{*} k_{\mathrm{B}} T / h e B$. The quantum mobility is then obtained from the so-called Dingle plots, i.e. plots where $\sinh (X) / X \Delta \rho_{x x}$ is plotted as function of $1 / B$.

\section{Results}

In Table 1 we present the results of the transport mobility measurements which where obtained from the classical part of the magneto-resistance measurements by the analysis method of Beck and Anderson [2]. Their analysis method could only be used in the low-doping concentration regime because we were not able to obtain reproducible results when three or more subbands are populated. Our results show a better mobility in the higher subbands. It seems reasonable to explain this by the smaller overlap of the wave function with the ionized impurities in the higher subbands, see Fig. 1.

In Table 2 we present the results of the determination of the quantum mobility in samples where the doping layer thickness was $2 \mathrm{~nm}$ or less. Firstly it is clear that the mobility increases with the subband number. Again the
Table 1

Transport mobility $\left(\mathrm{cm}^{2} / \mathrm{Vs}\right)$ in the populated subbands

\begin{tabular}{lrr}
\hline$N_{\text {DON }}\left(10^{12} \mathrm{~cm}^{-2}\right)$ & 2.0 & 3.5 \\
$N_{2 \mathrm{D}}\left(10^{12} \mathrm{~cm}^{-2}\right)$ & 1.8 & 3.2 \\
$l=0$ & 1500 & 1250 \\
$l=1$ & 7100 & 4800 \\
\hline
\end{tabular}

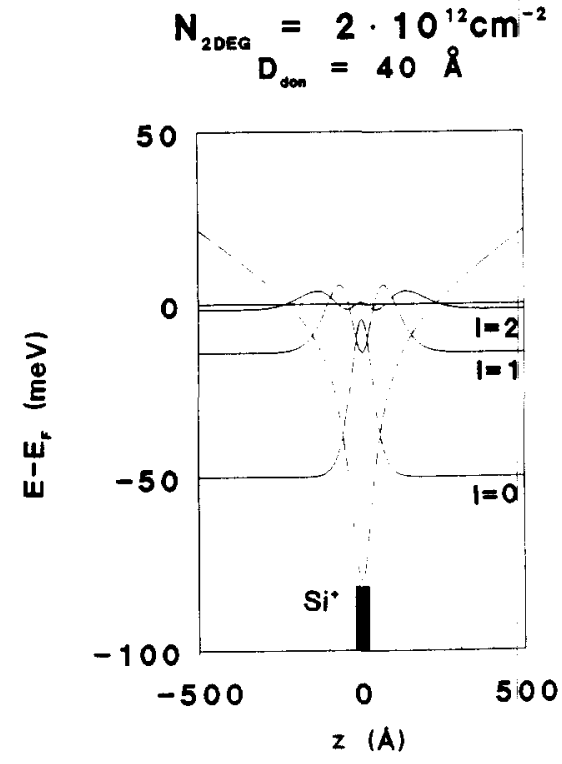

Fig. 1. Charge probability distribution in Si delta idoped GaAs.

Table 2

Quantum mobility $\left(\mathrm{cm}^{2} / \mathrm{Vs}\right)$ in the populated subbands

\begin{tabular}{lrrrrr}
\hline$N_{\text {DON }}\left(10^{12} \mathrm{~cm}^{-2}\right)$ & 2.0 & 2.5 & 3.5 & 5.0 & 8.0 \\
$N_{2 \mathbf{D}}\left(10^{12} \mathrm{~cm}^{-2}\right)$ & 1.8 & 2.2 & 3.2 & 4.5 & 5.6 \\
$l=0$ & 670 & 582 & 500 & 463 & 370 \\
$l=1$ & 4400 & 3390 & 2040 & 2020 & 1640 \\
$l=2$ & - & - & $\cdots$ & 4080 & 2450 \\
\hline
\end{tabular}

lowest subband, which is populated most heavily, has the lowest mobility. In the higher subbands the mobility is better. The table also clearly shows that the mobility decreases as function of the doping concentration. This drop might be due to an increasing overlap between scatterers and electron gas as a results of the stronger confining potential at higher doping concentrations.

The ratio of the transport to the quantum mobility is about a factor of two. This agrees with the results of Das Sarma et al. [2], who calculated that the ratio should be 


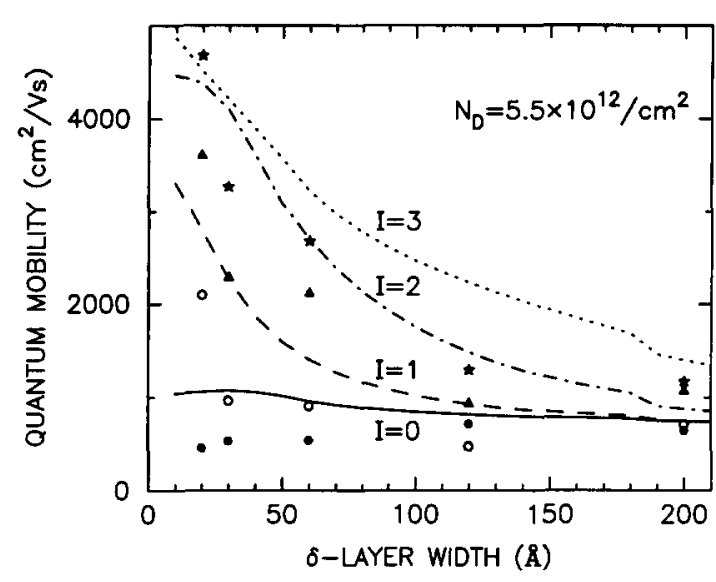

Fig. 2. Quantum mobility as a function of the doping layer thickness. The lines show the calculated quantum mobilities.

close to 2.5 for $\mathrm{GaAs} / \mathrm{AlGaAs}$ heterostructures where the ionized doping atoms overlap with the electrongas.

The sample with an electron concentration of $5.5 \times 10^{12} \mathrm{~cm}^{-2}$ was annealed at $800^{\circ} \mathrm{C}$ for various durations ranging from 3 to $300 \mathrm{~s}$. Due to the annealing the thickness of the doping layer increased from 2 to $20 \mathrm{~nm}$. The doping layer width was determined from a fit of the measured population with the calculated population where the width of the doping layer was the fitting parameter. We observed that the width could be described by a normal diffusion process, $\sigma=(2 D t)^{1 / 2}$. In Fig. 2 the mobility as a function of the thickness of the doping layer is plotted. The results show for an increasing thickness of the doping layer that the mobility drops to the same value for all subbands and that the mobility in the lowest subband is nearly independent on the doping layer thickness. Also in this case the overlap of scatterers and electron gas seems to be the main reason for the mobility drop in the higher subbands.

\section{Theory}

Tne mobility in the individual subbands has been calculated by Mezrin and Shik [3]. They calculated the transport mobility in the populated subbands for delta doped structures with different doping concentrations having a mathematical delta doping layer of zero thickness. If we compare their quantum mobility in the lowest subband with our experimental results for the structures with different doping concentrations we get a perfect fit. However their results did not show the quantum mobility in the higher subbands.

We have also calculated [4] the quantum and transport mobility. We used wave functions that were ob-

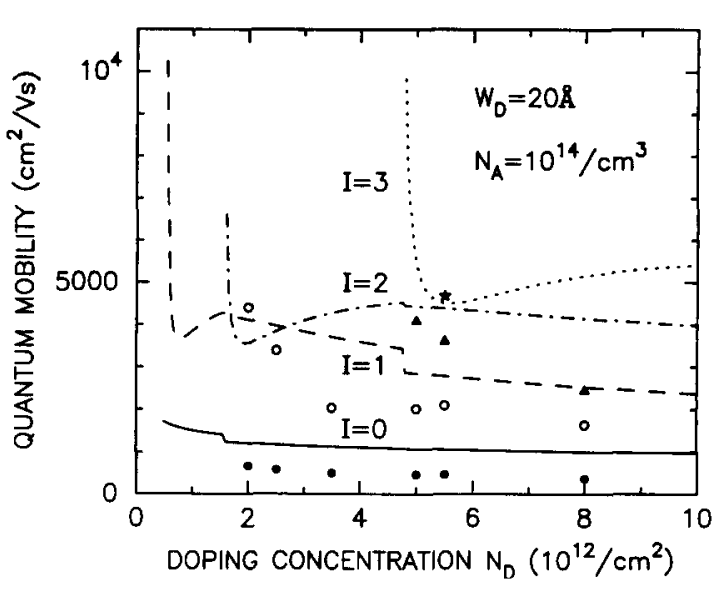

Fig. 3. Quantum mobility as a function of the doping concentration. The lines show the calculated quantum mobilities.

tained from the self-consistent solution of the Poisson and Schrödinger equations. Although we observe a very good fit for the lowest subband, in accordance with results of Mezrin and Shik, we find that the quantum mobility in the higher subbands is nearly equal to the mobility in the $i=0$ subband.

In studies of the mobility in GaAs/AlGaAs heterostructures it was proposed by several authors [5-7] that ionized impurity scattering is not determined by the full distribution of the ionized impurities but by the fluctuations in this distribution. Following these arguments the mobility can increase strongly. The reason for taking the fluctuations in the charge distribution lies in the fact that when the confined states are calculated, by solving the coupled Poisson and Schrödinger equations, the averaged distribution of the charged impurities is used. Thus when the scattering is calculated on the same impurity distribution each ionized doping atom would be used twice. In Figs. 2 and 3 we show the calculated mobility we have obtained by taking the fluctuations in the charge distribution as the scattering potential. In contrast with the calculation on the full distribution we now have a better mobility in the higher subbands. Overall we obtain a satisfactory agreement between the experimental results and the calculated mobilities.

\section{Conclusions}

Measurements of the quantum and transport mobility show that the ionized impurity scattering rate is determined by the overlap of the ionized impurities and the electron gas. The overlap decreases with the subband number leading to a better mobility in a higher subband. 
In an individual subband the overlap increases with the doping concentration resulting in a lower mobility at higher doping concentrations. By increasing the thickness of the doping layer the mobility in all subbands reduces to the mobility in the lowest subband due to strong overlap in all subbands. Comparison with mobility calculations shows that the scattering rate for ionized impurities is determined by the fluctuations in the charge distribution of the delta doping layer instead of the full charge distribution.

\section{Acknowledgements}

JD acknowledges the sponsorship of the Belgian National Science Foundation project no 2.0093.91.

\section{References}

[1] S. Das Sarma and F. Stern, Phys. Rev. B 32 (1985) 8442.

[2] W.A. Beck and J.R. Anderson, J. Appl. Phys. 62 (1987) 541.

[3] O.A. Mezrin and A.Y. Shik, Superlatt. Microstruct. 10 (1990) 107; O.A. Mezrin, A.Y. Shik and V.O. Mezrin, Semicond. Sci. Technol. 7 (1992) 664.

[4] G.Q. Hai, N. Studart, F.M. Peeters, J.T. Devreese, P.M. Koenraad, A.F.W. van de Stadt and J.H. Wolter, in: Acc. Int. Conf. Physics of Semiconductors, August, 1994, Vancouver, Canada.

[5] P.J. van Hall, T. Klaver and J.H. Wolter, Semicond. Sci. Technol 3 (1988) 120.

[6] R. Lassnig, Solid State Commun. 65 (1988) 765.

[7] E.F. Schubert, L. Pfeiffer, K.W. West and A. Izabell, Appl. Phys. Lett. 54 (1989) 1350. 